\title{
Damaged Number Plate Recognition
}

\author{
P. Ezhilarasi, S. Rajeshkannan, Kovendan. A K P, T. Sasilatha, K. R. Kayalvizhi
}

\begin{abstract}
Around the world every vehicle are identified by its number plate. Number plate detection is one of the existing automated video surveillance systems that are used to detect the number plate. This system fails if the number plates are damaged, no proper illumination, blurry images. Thus here we will be able to recognizeze such damaged number plate. The technique involves four main stages viz. pre-processing, localization, recognition and segmentation. The entire process includes capturing the image, erasing the background details and removing the noise, cropping the number plate and then recognizing the characters followed by segmenting in order to recognize the plate. All this is done in Python because it had better results compared to MATLAB. When done in MATLAB, additional error and noise gets added to the input image and can causes inclusion of a new characters in the number plate and leads to misinterpretation of the number plate. About 100 images were gathered and 98 images of them were detected correctly. The efficiency in recognizing the damaged number plate using our system is about $98 \%$.
\end{abstract}

Keywords: Pre-Processing; Character Segmentation; Character Recognition; Localization.

\section{INTRODUCTION}

With increasing population, the number of vehicles on roads is also increasing and thus it is very difficult to enforce laws and traffic rules manually for smooth traffic flow. Traffic management systems are installed on freeways to check for vehicles moving at speeds not permitted by law. All these processes have a scope of improvement. In the centre of all these systems is a vehicle. In order to automate these processes and make them more effective, a system is required to easily identify a vehicle. Thus automatic number plate recognition system (ANPR) was introduced, where many contributed their ideas to detect the number plate. plate can be recognised under any circumstances such as poor illumination, bad weather. The question is "What if the number plate is damaged?"

Existing system used neural network to describe the design algorithm but at the same time it will require a huge database [1]. Similarly convolutional neural network was used for the process but this technique was applicable only for Indian plate [2].

Revised Manuscript Received on December 22, 2019.

P. Ezhilarasi, Department of ECE, St. Joseph's College of Engineering, OMR, Chennai 119

S. Rajeshkannan, Department of ECE, St. Joseph's College of Engineering, OMR, Chennai 119

Kovendan. A K P, Department of ECE, St. Joseph's College of Engineering, OMR, Chennai 119

T. Sasilatha, Professor and Dean, Department of EEE, AMET Deemed to be University, Chennai

K. R. Kayalvizhi, Department of ECE, St. Joseph's College of Engineering, OMR, Chennai 119 Technology has improved in such a way that the number

A reliable approach was proposed using mathematical morphology and Support Vector Machine (SVM).This global approach for binarization had variations in illumination [3]. License Plates Recognition (LPR) is an Intelligent Transportation System (ITS) which gives specific information like owner of the vehicle, place of registration, address of the owner, etc and this information can be used by the military and defence officers for surveillance purpose. But the camera used in this system was very sensitive to vibration and fast changing targets near Line of Control (LoC) [5]. Certain work involves investigation into real-time ANPR and its extension for traffic monitoring and control. This system at times couldn't bring the number plate within the camera angle view and was unable to discriminate between D and O, C and G [4]. Further development was implemented using FPGA realization of the pre-processing stage which works on morphological approach and improved them using adaptive technique where the threshold level varies according to the input image. The background details, radiator grille lead to false detection [6]. Multiple plates were recognized and SCANMP algorithm was used for plate segmentation which eliminated noisy objects and reduced the processing time. Aspect ratio was used as the base for detection, now what if the number plate is damaged and the ratio is changed the probability of character loss during validation is possible [7]. To locate the license plate at the right position, multi Cluster approach was used and to extract features from the license plates, Kirsch Edge feature extraction technique was applied. These lead to more time for classification and also error occurred in differentiating between B and 3, 5 and 6, 6 and G, A and 4 and I to 1 [8]. M. J. Ahmed et.al. [9] Proposed the Automated LPR system for Saudi Arabia which had several unique features to be considered and matching template is to be used for recognition based on hamming distance between the characters. During the acquisition stage, the quality of input image should be taken care as it is the only reason for misrecognition. Musab Mohammed Bagabir et.al. [10] Presented a framework for Sudanese VLPR system. They used MOPSO (Multi Objective Particle Swarm Optimization) which aimed to produce good segmentation results. The method depends on skew. The skew correction depends on finding the angle of rotation, and then the extracted license plate image is rotated using that angle of rotation. Accuracy lies in correct identification of the skew. The side lines of the plate for License Plate Location Detection (LPLD) was detected by Hough transform. The advantage of this transform is that it detects lines and curves efficiently, but the time taken for plate detection is very high. 


\section{PROPOSED WORK}

The proposed work is aimed to detect damaged number plate and recognize them. It involves four main stages which include:

1) Pre-processing

2) License plate localization

3) Character recognition

4) Character segmentation

The system deals with different angles, distances and illumination conditions.

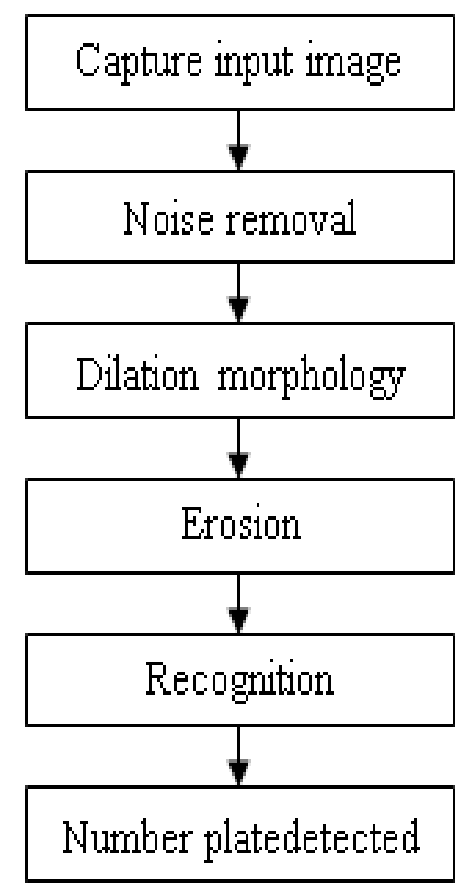

Fig. 1 Main steps of LPR

\section{Pre-processing}

Pre-processing is done to enhance the input image and making it more suitable for the next processing stages. Thus the minimum filter is applied to the image in order to enhance the dark values in the image by increasing their area. This process is done to make the plate edges and the characters bold, and also to remove the effect of the light diagonal strips that appear in edges of the license plates and the characters.

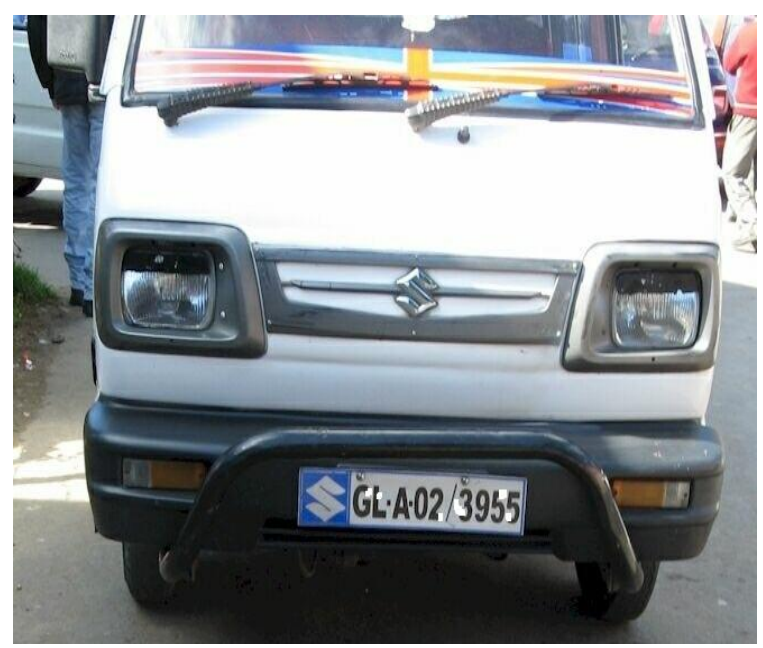

Fig. 2 Before Sobel edge detection applied
Image acquisition, images of the number plate are obtained using a camera. The obtained colour image is converted into a grey scale by using weighted sum method

$$
\mathrm{I}_{\mathrm{G}}=0.2989 * \mathrm{R}+0.578 * \mathrm{G}+0.114 * \mathrm{~B}
$$

Image binarizaion, using a suitable threshold value grey scale images are converted into binary images for better output. Hence the resultant image has only black and white pixels. The variable size image gets transformed into a binary matrix of fixed pre-determined dimensions in order to achieve uniformity in the dimensions of the input which leads to reduction in computation time.

During this process only, the Gaussian noise is removed and the contours are marked to get the license plate. Also background noise is removed.

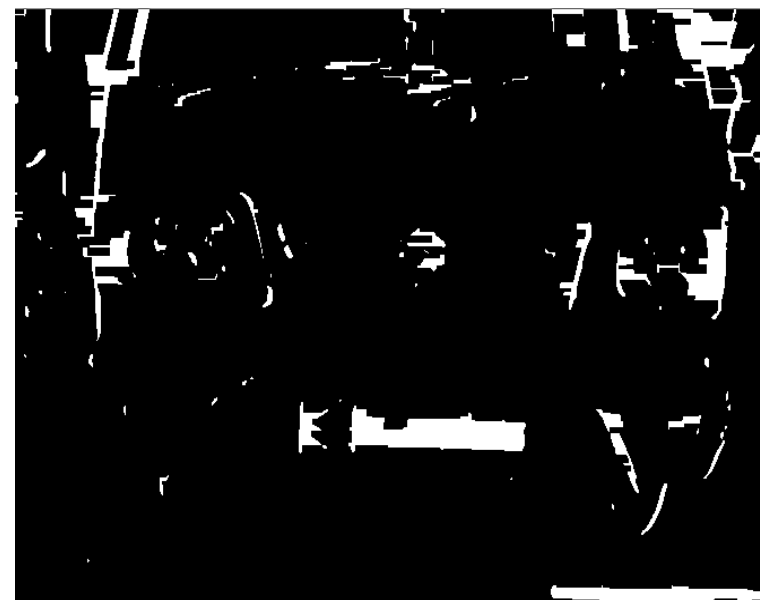

Fig. 3 After Sobel edge detection applied

\section{License plate localization}

The accuracy of the system is been developed by this process. In a given image, the goal of this phase is to produce a number of regions with high probability of containing a license plate. The contours will be helpful in detecting the license plate based on the threshold. The contours marked will be concentrating the license plate alone.

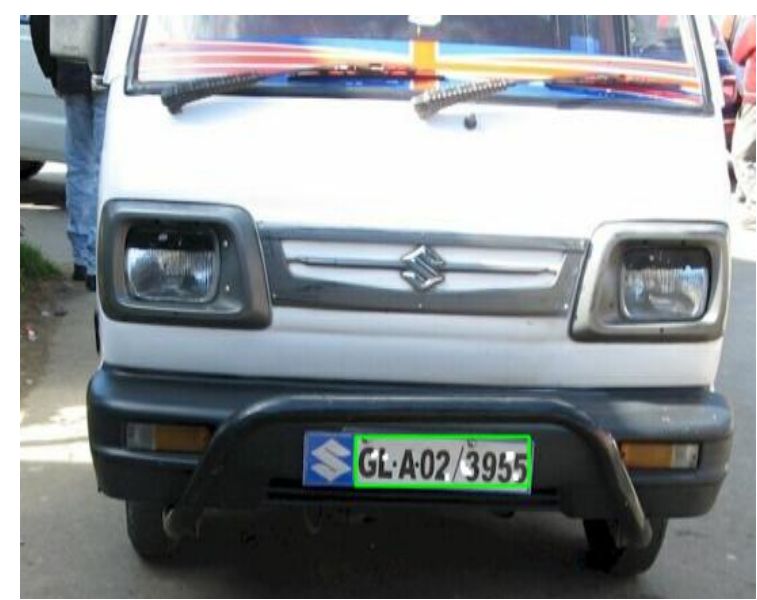

Fig. 4 Afterlocalization of number plate

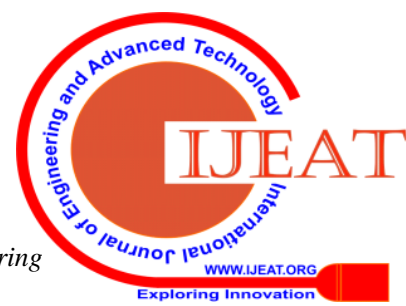


If suppose the number plate is damaged or rotated, by checking the aspect ratio using height and width of the number plate ,the number plate can be perfectly localized.

This is done by the classification of the vehicle from which the license plate is extracted. The contours marked will help in cropping the license plate, removing other details surrounding the license plate. The damaged license plate will be the output at this stage.

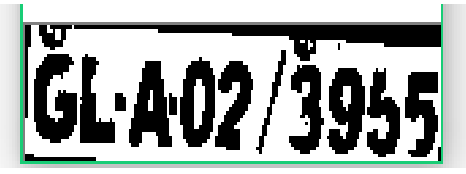

Fig. 5 After cropping

\section{Character Recognition}

The cropped license plate will now be used to recognize the characters in the plate. Number plate's characters are ratio is found then the characters present in the plate can be recognised. Once the characters are found it will be compared with other characters in order to identify it. This includes the Morphological techniques viz. Dilation and lacks the proper shape because of the damage to it. Once the pixels are added then erosion is performed where edges are drawn around the character to mark it. After the marking they will be compared with a set of templates which will report the matched character. During this the characters will be rotated in order to find the exact character that matches the characters saved in the library.

\section{Character Segmentation}

The first and foremost process in the recognition of number plate is segmentation because all other auxiliary phases depend on this process. A character can be estranged into dual portions, or it will look like two characters when segmentation fails. Hence the definitive elucidation for this badly-behaved is to use bounding box technique. The properties of the image region are measured using bounding box. When a bounding box is created for every set of numbers and character obtainable on number plate, each number and character is isolated for recognition of number plate.

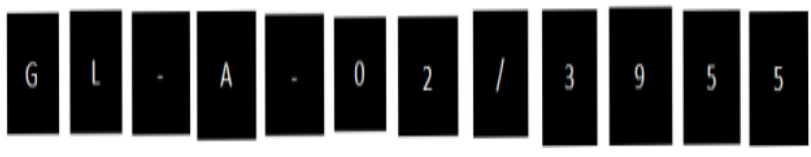

Fig. 6 Image after segmentation process

\section{EXPERIMENTAL ANALYSIS}

Damaged number plates were fed as input to the system, processes took place and final output of the detected number plate was displayed. The efficiency of the system with other systems is shown in the table below:

Table. 1 Efficiency of the system

\begin{tabular}{|l|c|c|}
\hline Software & Input Images & Efficiency \\
\hline Matlab & 750 & $92 \%$ \\
\hline
\end{tabular}
found in the plate according to the aspect ratio. If the aspect Erosion. In dilation, pixels are added to the character which

\begin{tabular}{|l|l|l|}
\hline OpenCV (Python) & 100 & $98 \%$ \\
\hline
\end{tabular}

The accuracy of the system during the whole process is shown below in table 2 .

Table. 2 Results of damaged LP detection and recognition

\begin{tabular}{|c|c|c|}
\hline $\begin{array}{c}\text { LP Detection } \\
\text { Accuracy }\end{array}$ & $\begin{array}{c}\text { LP Identification } \\
\text { Accuracy }\end{array}$ & $\begin{array}{c}\text { Recognition } \\
\text { Accuracy }\end{array}$ \\
\hline $97 \%$ & $98 \%$ & $97 \%$ \\
\hline
\end{tabular}

\section{Applications}

I. Access control:-In a secured area, gate will be opened automatically for authorized members, thus substituting or supporting the security guard.

II. Tolling: Instead of faster registration number of the car can be used to charge the travel fee by recognition of the number plate in a toll-road or used to check the ticket twice.

III. Border Security:-The car number plate can be recognized and the number can be registered for either the entry or exits to the country and it is used to monitor the border crossings.

IV. Parking:-The number plate recognition unit is used to enter prepaid members automatically and calculate parking fee for nonmembers.

V. Traffic Control:-The vehicles can be engaged to dissimilar ways rendering their entry permits. Hence the traffic congestions and number of attendants can be reduced.

VI. Airport Parking:-Ticket forgery or mistakes can be reduced by capturing the number plate images of the car with the help of number plate recognition unit.

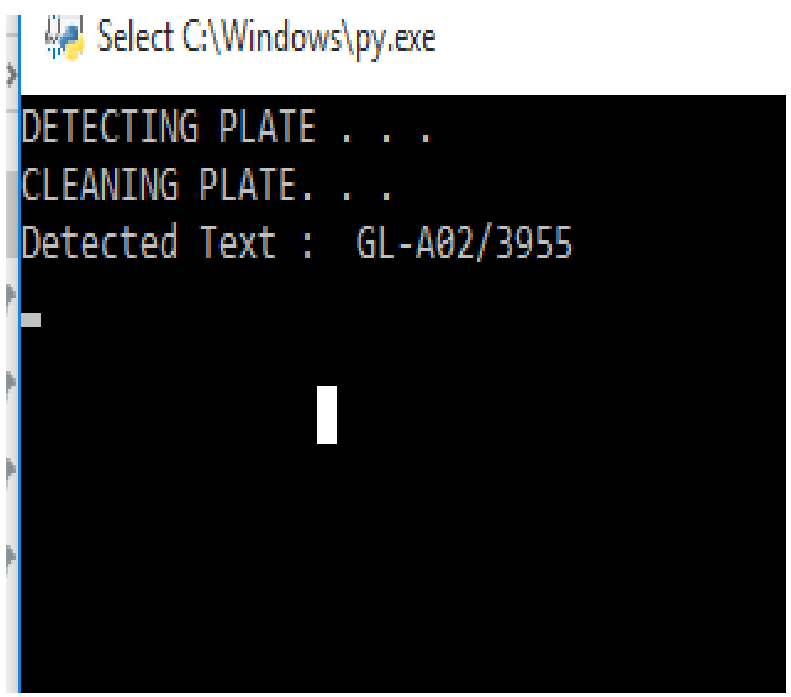

Fig. 7 Image of result

\section{CONCLUSION}

To detect the damaged number plate of vehicles, here an application software has been designed, even if the number plate is damaged, broken, with low resolution or blurred image. This application may be linked with the RTO office to have a database that records the vehicle which are having damaged number plate. It will be helpful in video surveillance applications.

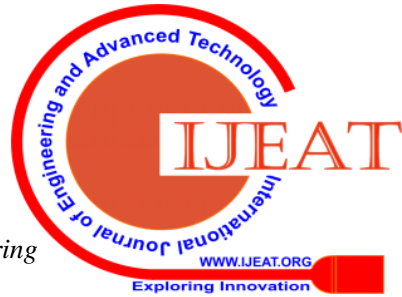




\section{REFERENCES}

1. Ragini Bhat and Bijender Mehandia, "Recognition of Vehicle Number Plate using MATLAB", International Journal of Innovative Research in Electrical, Electronics, Instrumentation and Control engineering Vol.2,Issue 8,August 2014.

2. Dening Jiang, Tulu Muluneh Mekonnen, Tiruneh Embiale Merkebu and Ashenafi Gebrehiwot,"Car Plate Recognition System",2012 Fifth International Conference on Intelligent Networks and Intelligent Systems.

3. Surajit Das and Joydeep Mukherje, "Automatic License Plate Recognition Technique using Convolutional Neural Network" International Journal of Computer Applications(0975-8887) -No.4,July 2017.

4. Ayman Rabee and Imad Barhumi,"License Plate Detection and Recognition in Complex scenes using Mathematical Morphology and support vector machines", International Conference on Systems, Signals and Image Processing,12-15 May 2014.

5. Muhammad TahirQadri and Muhammad Asif,"Automatic Number Plate Recognition System using Optical Character Recognition", IEEE-2009 International Conference on Education Technology and Computer.

6. M. Ashouriana, N. Daneshmandpoura, O. Sharifi Tehrania , P. Moallemb, "Real Time Implementation of a License Plate Location Recognition System Based on Adaptive Morphology",IJE TRANSACTIONS B: Applications Vol. 26, No. 11, (November 2013) 1347-1356.

7. Nabeel Younus Khan II, Ali Shariq Imranand Naveed Ali, "Distance and Color Invariant Automatic License Plate Recognition System" (2007 IEEE).

8. Siti Norul Huda Sheikh Abdullah, Marzuki Khalid and Rubiyah Yusof,“ License Plate Recognition using Multi-cluster and Multilayer Neural Networks", (IEEE 2006).

9. M J.Ahmed, M Sarfaz, A. Zidouri and KG .AI Khatib ,"License plate Recognition System"(IEEE-2003).

10. Sujatha, V., Mary Anita, E.A., "Fuzzy based scheme for detection of Sybil node in wireless sensor networks" Journal of Advanced Research in Dynamical and Control Systems, 2017

11. Shanthi H.J., Mary Anita E.A, "Secure and efficient distance effect routing algorithm for mobility (SE_DREAM) in MANETs" Smart Innovation, Systems and Technologies, 2016.

12. D Arivazhagan, S Sundaram, "Challenges of Cloud Computing, Security Issues and Potential Operational Areas in Data Transportability, Segregation, Backup, Portability and Recovery", International Journal of Emerging Technologies and Innovative Research, Volume 5, Issue 7, Pages: 681-683, 2018. 